\title{
Antiallodynic Effect of Hyperbaric Oxygen on Paclitaxel-Induced Peripheral Neuropathy in a Model of Rat and Its Mechanism
}

\author{
Ting Miao' ${ }^{1}$ Xianze Meng², Hong He ${ }^{3}$, Xiaojun $\mathrm{Ji}^{3^{*}}$ \\ ${ }^{1}$ Institute of Integrative Medicine, Qingdao University, Qingdao, China \\ ${ }^{2}$ Department of Traditional Chinese Medicine, No 971 Hospital of PLA, Qingdao, China \\ ${ }^{3}$ Affiliated Hospital of Qingdao University, Qingdao, China \\ Email: miaoting56@163.com, *jixiaojun2@medmail.com.cn
}

How to cite this paper: Miao, T., Meng, X.Z., He, H. and Ji, X.J. (2019) Antiallodynic Effect of Hyperbaric Oxygen on PaclitaxelInduced Peripheral Neuropathy in a Model of Rat and Its Mechanism. Open Access Library Journal, 6: e5339.

https://doi.org/10.4236/oalib.1105339

Received: March 15, 2019

Accepted: April 9, 2019

Published: April 12, 2019

Copyright $\odot 2019$ by author(s) and Open Access Library Inc.

This work is licensed under the Creative Commons Attribution International License (CC BY 4.0).

http://creativecommons.org/licenses/by/4.0/

\section{Open Access}

\begin{abstract}
Objective: To investigate the antiallodynic effect of Hyperbaric oxygen treatment (HBOT) on paclitaxel (PTX)-induced peripheral neuropathy in a model of rat and the influence on Astrocyte activation-Inflammatory pathway. Method: 45 male Sprague-Dawley rats were equally divided into 3 groups, the Control group (Control), the Model group (PTX), the Therapy group (PTX + HBOT). Except for the Control group, all groups were intraperitoneally injected with paclitaxel $2 \mathrm{mg} / \mathrm{kg}$ on day $1,3,5,7$, respectively, and a stable peripheral neuralgia model was established on the 14th day. The Therapy group was treated with HBOT on 2.5 ATA for 7 days after modeling. We used the UP and Down method to test the mechanical withdraw threshold (MWT) of rats before and after modeling, during and after treatment. One week after the end of treatment, each group of rats was taken spinal cord L4-L6 for subsequent molecular biology experiments. The expression and location of astrocyte activation marker GFAP in spinal cord were assayed by Immunohistochemistry. The expression of GFAP, TNF- $\alpha$, IL- $1 \beta$ was assayed by western blot analysis. The expression of mRNA of GFAP, TNF- $\alpha$, IL- $1 \beta$ was assayed by realtime-RT PCR. Results: On the nineteenth day of the experiment (the fifth day of treatment), the MWT of Treatment group was significantly higher than that of Model group $(\mathrm{p}<0.001)$. This effect persisted for one week after the end of treatment (day $21-28, \mathrm{p}<0.001$ ). Immunohistochemistry showed that the expression of GFAP was significantly lower in Treatment group than in Model group $(\mathrm{p}<0.01)$. Western Blot Analysis showed that the expression of GFAP, IL- $1 \beta$, TNF- $\alpha$ was significantly lower in Treatment group than in Model group $(\mathrm{p}<0.05, \mathrm{p}<0.01, \mathrm{p}<0.01)$. Realtime-RT PCR showed that the expression of mRNA levels of GFAP was
\end{abstract}


slightly higher in Model group than in Control group ( $\mathrm{p}=0.0467)$, but there was no statistical difference between the two groups. The expression of mRNA of IL- $1 \beta$, TNF- $\alpha$ was significantly lower in Treatment group than in Model group $(\mathrm{p}<0.01, \mathrm{p}<0.01)$. Conclusion: Hyperbaric oxygen treatment alleviates paclitaxel-induced peripheral neuralgia which has a long-term therapeutic effect, and inhibits the activation of spinal astrocytes and the level of inflammatory cytokines for neuroinflammation.

\section{Subject Areas}

Internal Medicine

\section{Keywords}

Hyperbaric Oxygen, Paclitaxel, Peripheral Neuropathy, Astrocyte, Interleukin, Rats

\section{Introduction}

Chemotherapy-induced peripheral neuropathic pain (CIPNP) is the main side effect of the use of purple alkanes, platinum and vinblastine. Surveys have shown that $80 \%$ of patients have this side effect in routine chemotherapy [1]. The main symptoms are numbness and tingling sensation in extremities, and sometimes presenting spontaneous pain, autonomic nerve dysfunctions [2]. Although some symptoms can be relieved within a few months after chemotherapy, many patients will turn to chronic pain, seriously affecting the quality of living [3]. There is a dose-dependent relationship between pain and chemotherapy. Many cannot tolerate and change the chemotherapy plan, or even give up. At present, the first-line medication for CIPNP is mainly antidepressants, anticonvulsants and opioids, but has limited analgesic effect with great side effects [4]. Therefore, searching for methods with safety and efficacy on treatment of CIPNP has become a hotspot.

Hyperbaric oxygen (HBOT) therapy is pure oxygen that patients breathe at more than one atmospheric pressure in the oxygen chamber. It can improve tissue oxygen pressure, treating multiple injuries, $\mathrm{CO}$ poisoning, decompression sickness, etc. It is a cheap and minimal side-effect, which is more acceptable. A growing number of reports showed that HBOT can relieve kinds of chronic pain [5]. But for CIPNP, none has been studied. The pathogenesis of CIPNP is very complex, and the multiple mechanisms depend on different chemotherapeutic drugs [1], but all of them are concerning central sensitization caused by neuroinflammation mediated by spinal astrocyte activation [1] [6]. Some studies showed that the use of glial cell regulators can alleviate paclitaxel-induced thermo algesia [7], and the activation of glial cells is also related to the persistence of pain [8] [9]. HBOT can mediate anti-inflammatory and analgesic effects by regulating the activation of spinal astrocytes [10] [11] [12]. However, current 
studies have not involved CIPNP and have not revealed the long-term analgesic effects of HBOT on neuroinflammation. The aim of this study is to confirm the antiallodynic effect of HBOT on CIPNP in a model of rat associated with neuroinflammation.

\section{Methods and Materials}

\subsection{Animals}

Forty-five healthy adult male Sprague-Dawley rats, aged 8 weeks and weighting 275 - 325 g, were provided by Jinan Pengyue Laboratory Animal Breeding Co. China, Ltd. [SCXK (Lu) 2014 0007]. The animals were housed individually in plastic boxes at $23^{\circ} \mathrm{C}\left( \pm 0.5^{\circ} \mathrm{C}\right)$, drinking and eating freely. The Animal Ethics Committee of Qingdao University (Qingdao, China) approved all protocols for the Care and Use of Laboratory Animals.

\subsection{Experimental Protocol}

$45 \mathrm{SD}$ rats were randomly divided into three groups, 15 in each. They were Control group, Model group (PTX) and Treatment group (PTX + HBOT), respectly. Rats in the treatment group and model group were intraperitoneally injected with paclitaxel $2 \mathrm{mg} / \mathrm{kg} / \mathrm{ml}$ (paclitaxel dissolved in ethanol:cremophor EL:saline $=1: 1: 4)$ on the 1st, 3rd, 5th and 7th days of the experiment, and a stable peripheral neuralgia model could be established on the 14th day [13]. The control group was given intraperitoneal injection of solvent $1 \mathrm{ml} / \mathrm{kg}$ (ethanol:cremophor EL:saline $=1: 1: 4$ ).

An animal HBOT chamber (YSD-I; Yantai Binglun Oxygen Industry Co., Ltd., Shandong, China) was utilized for HBOT treatment. 100\% pure oxygen was filled with cabin, and quick lime was placed to further absorb $\mathrm{CO}_{2}$ and water vapor. Animals in the Treatment group were then placed into the chamber. The pressure within was gradually increased at a rate of $0.1 \mathrm{ATA} / \mathrm{min}$ until 2.5 ATA, and was maintained for one hour. At the end of the therapy, the pressure was gradually decompressed to atmospheric pressure at a rate of $0.1 \mathrm{ATA} / \mathrm{min}$ [10]. HBOT treatment lasted for 7 consecutive days (day 15 - day 21). Animals in model group were allowed to stay in the chamber without any treatment.

After treatment, an additional 7 days (day 22 - day 28) were to observe long-term effect on anti-allodynic and then rats were sacrificed for subsequent tests.

\subsection{Behavioral Analysis}

Rat mechanical withdrawal threshold (MWT, Mechanical Withdraw Threshold) was tested using Von Frey fiber (Stoelting Co.) by Up and Down method [14]. Stimulating the plantar surface of the hind paw for $3-5 \mathrm{~s}$ with an interval of 30 $\mathrm{s}$, a withdrawal response was described as positive and turned to a lower force. In contrast, a higher force was conducted when a negative response occurred. 1 g, 1.4 g, 2 g, 4 g, 6 g, 8 g, 10 g, 15 g, eight Von Fery fibers were selected according 
to preliminary experiment, which started from $4 \mathrm{~g}$ and cut off at $15 \mathrm{~g}$. The rats were tested 6 times after the first positive response, and finally the MWT ( $g$ ) was calculated based on the arrangement of the positive and negative patterns. The MWT values were measured before modeling and during the treatment (Day 15, Day 17, Day 19, Day 21) and after treatment for observing long-term efficacy (Day 24, Day 28).

\subsection{Immunohistochemistry}

To examine astrocyte profiles on the long-term antiallodynic effect, five rats in each group were anesthetized with $10 \%$ chloral hydrate at $28^{\text {th }}$ day after behavioral tests, then the spinal cord L4-L6 were taken and fixed in $4 \%$ paraformaldehyde in $0.1 \mathrm{M}$ phosphate buffer (PBS), $\mathrm{pH} 7.4$ overnight at $4^{\circ} \mathrm{C}$. After fixation, $5-7 \mu \mathrm{m}$ sections of spinal cord were cut and washed in PBS and then incubated with $3 \% \mathrm{H}_{2} \mathrm{O}_{2}$ to block peroxidase. They were washed in PBS three times and pre-incubated for $30 \mathrm{~min}$ at room temperature with $10 \%$ goat serum in PBS containing $0.7 \%$ Triton $\mathrm{X}-100$. The sections were incubated overnight at $4^{\circ} \mathrm{C}$ with rabbit anti-glial fibrillary acidic protein (GFAP) primary antibody [1:200] (Absin CO., China) and then after rinsing in PBS $(3 \times 10 \mathrm{~min})$, incubated with secondary HRP-conjugated antibody for $30 \mathrm{~min}$ at room temperature. The substrate 3,30-diaminobenzidine (DAB) was applied for $5 \mathrm{~min}$, and then were counterstained with hematoxylin. The signals of each group were normalized to Control group as fold increase for further statistical analysis using Image-Pro Plus Version 6.0.

\subsection{Western Blot}

To examine the expression levels of related proteins on the long-term antiallodynic effect, the spinal cords were taken from five rats as described above and kept fresh on the ice. Proteins were extracted by RIPA lysis buffer (p0013C, Beyotime, China), and then heated at $95^{\circ} \mathrm{C}$ with loading buffer for 5 minutes for denaturation. $20 \mu \mathrm{g}$ denaturated proteins for each were separated by $10 \%$ SDS-PAGE and then transferred onto a polyvinylidene difluoride (PVDF) membrane. After blocking for 1 hour at room temperature in Tris-buffered saline containing 5\% non-fat milk, the membranes were incubated overnight at $4^{\circ} \mathrm{C}$ with the primary antibody Rabbit anti-GFAP (Glial fibrillary acidic protein) polyclonal antibody (1:500; absin131715, Absin CO., China), Rabbit Anti-TNF alpha Polyclonal Antibody (1:500; absin123966, Absin CO., China), Rabbit Anti-IL-1 Beta Polyclonal Antibody (1:500; absin126104, Absin CO., China), Rabbit anti- $\beta$-actin monoclonal antibody (1:1000; absin132001, Absin CO., China). Following washes in TBST buffer, HRP-conjugated secondary antibody (1:2000; absin20002, Absin CO., China) was used to detect primary antibodies at room temperature for 2 hours. Peroxidase activity was visualized by ECL plus reagent and capture images. The intensity signals of each protein were normalized by that of $\beta$-actin and changes between groups were expressed as fold increase of other groups versus the control group. 


\subsection{Real-Time PCR}

To examine the expression levels of related mRNA on the long-term antiallodynic effect, the spinal cords were taken from five rats as described above and kept fresh on the ice. Total RNA was extracted according to the instructions of Trizol (R1100, Solarbio CO.) and the cDNA were obtained according to the instructions of the Reverse Transcription Kit (RP1105, Solarbio CO.). SYBR Green PCR kit (SR1120, Solarbio CO.) was used for PCR amplification and quantitative analysis. The common amplification conditions were set as: $95^{\circ} \mathrm{C} 30 \mathrm{~s}, 60^{\circ} \mathrm{C} 60 \mathrm{~s}$, $72^{\circ} \mathrm{C} 2 \mathrm{~min}, 45$ cycles. The specificity of primers was observed by dissolution curve, and the CT values were recorded. Statistical analysis was carried out by using $2^{-\Delta \Delta c t}$ [15], $\Delta \mathrm{CT}=\mathrm{CT}$ (target gene) $-\mathrm{CT}(\mathrm{GAPDH}), \Delta \Delta \mathrm{CT}=\Delta \mathrm{CT}($ target group) $-\Delta \mathrm{Ct}$ (mean value of $\Delta \mathrm{CT}$ in control group for describing changes between groups as fold increase ). All $\Delta \Delta \mathrm{CT}$ converted to $2^{-\Delta \Delta c t}$ for subsequent statistical analysis.

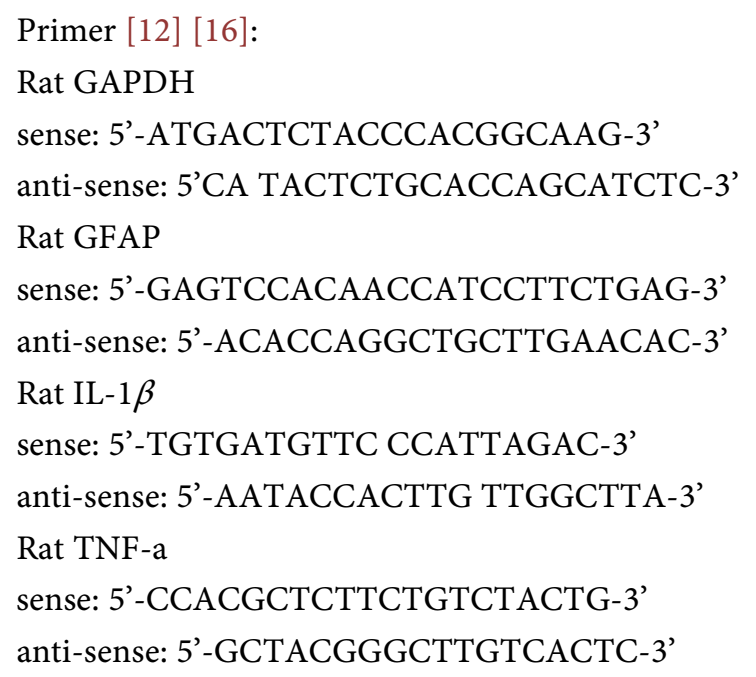

\subsection{Statistical Analysis}

Changes among groups in behavioral analysis and weight changes were compared using two-way repeated-measures analysis of variance (ANOVA) with post hoc Bonferroni tests. One-way ANOVA with post hoc Bonferroni correction was used to identify differences among groups in Immunohistochemistry and real-time RT-PCR. Data are presented as means \pm standard errors of the mean. P values $<0.05$ were considered significant. For real-time RT-PCR, P values $<0.01$ were considered significant.

\section{Result}

\subsection{Body Weight}

Body weight changes did not differ between paclitaxel- or solvent-treated animals ( $P>0.05$ Figure 1). There were no hair loss and diarrhea in all groups of rats during the experiment. It shows that the HBOT, paclitaxel and paclitaxel solvents have no influence on the health of rats under this experimental design. 
$\square$ PTX

$\ominus$ PTX+HBO
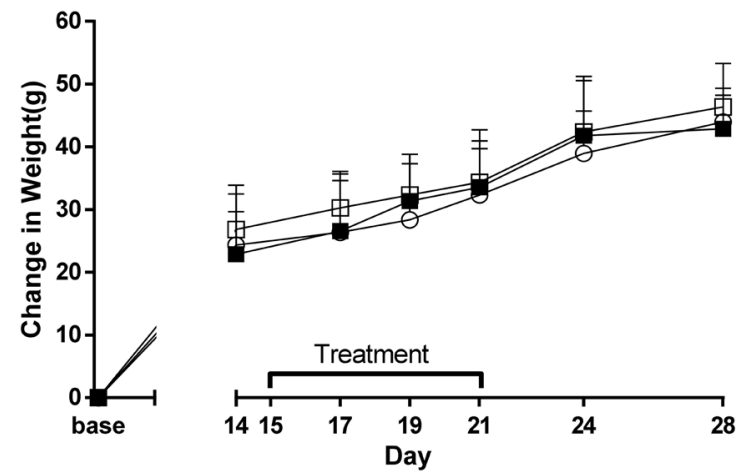

Figure 1. No changes in weight between groups throughout the experiment, $\mathrm{N}=15$ per group.

\subsection{Anti-Allodynic Effects of the HBOT}

On the 14th day after intraperitoneal injection of paclitaxel, the MWT value of the Control group was significantly lower than that of the Model group $(\mathrm{P}<$ 0.001 , Day 14, Figure 2), indicating that the paclitaxel can cause significant hyperalgesia. The MWT value of the Treatment group had an upward trend and from the 19th day (5th day of treatment) statistical difference had been observed ( $\mathrm{P}<0.001$, Day 19, Figure 2). The value maintained the level of the 19th day until the end of the treatment $(P<0.001$, Day 21, Figure 2$)$. After treatment, the value became to decrease, but still maintained a relative high level compared with Model group ( $<<0.001$, Day 24 Day 28, Figure 2$)$, indicating that the analgesic effect of $\mathrm{HBOT}$ was sustainable after the cessation of treatment.

\subsection{Inhibition of Astrocyte Activation}

The expression of GFAP in the Model group was significantly higher than that in the Control group $(\mathrm{P}<0.01$, Figure 3; $\mathrm{P}<0.01$, Figure $4(\mathrm{a})$ ), suggesting that paclitaxel can activate astrocytes. The Treatment group was significantly lower than that in the Model group ( $\mathrm{P}<0.01$, Figure 3; $\mathrm{P}<0.05$, Figure $4(\mathrm{a})$ ), indicating that HBOT inhibited the activation of astrocytes. The expression of mRNA levels of GFAP were no significant differences between groups $(\mathrm{P}=$ 0.0467 , Figure $5(a)$, size of test $p<0.01)$.

\subsection{Expression of IL-1 $\beta$ and TNF- $\alpha$ in the Spinal Cord}

The expression of IL-1 $\beta$ and TNF- $\alpha$ in the Model group was significantly higher than that in the Control group ( $\mathrm{P}<0.001$, Figure 4(b) and Figure 4(c)), indicating that paclitaxel can induce neuroinflammation. The expression of IL-1 $\beta$ and TNF- $\alpha$ in the Treatment group was significantly lower than that in the Model group ( $\mathrm{P}<0.01$, Figure 4(b) and Figure 4(c)), indicating that HBOT in- 
hibited neuroinflammation. The expression of mRNA levels of IL- $1 \beta$ and TNF- $\alpha$ was significantly increased in the model group than that of Control group $(\mathrm{P}<$ 0.001, Figure 5(b) and Figure 5(c)), indicating that paclitaxel up-regulated the mRNA levels of IL- $1 \beta$ and TNF- $\alpha$, resulting in a long-term neuroinflammation. The mRNA levels in Treatment group was significantly lower than that in the Model group $(\mathrm{P}<0.01$, Figure $5(\mathrm{~b})$ and Figure 5(c)), indicating that HBOT could reduce the mRNA levels of IL- $1 \beta$ and TNF- $\alpha$ producing a long-term analgesic effect.

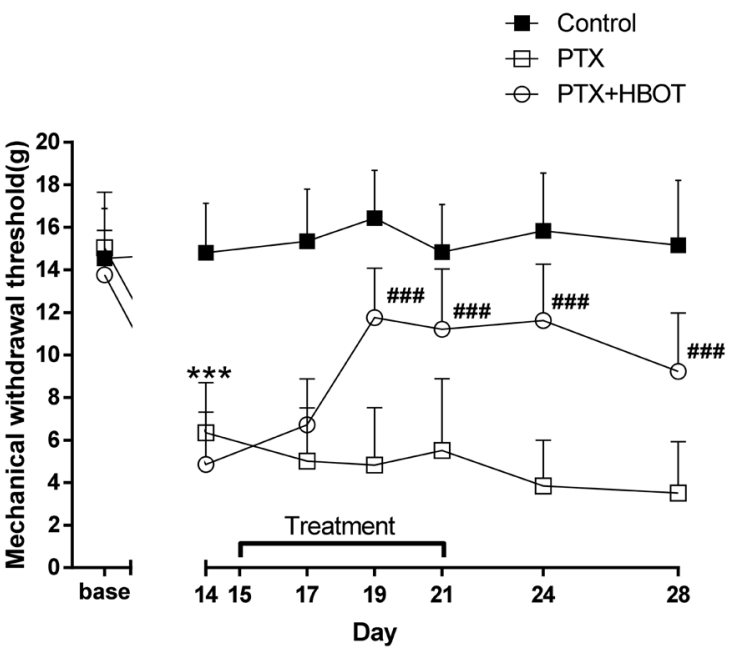

Figure 2. HBOT produced a long antinociceptive effect affecting the mechanical withdrawal threshold (MWT). $\mathrm{N}=15$ per group. ${ }^{* * *} \mathrm{P}<0.001$ vs. Control group, ${ }^{\# \#} \mathrm{P}<0.05$ vs. PTX group.
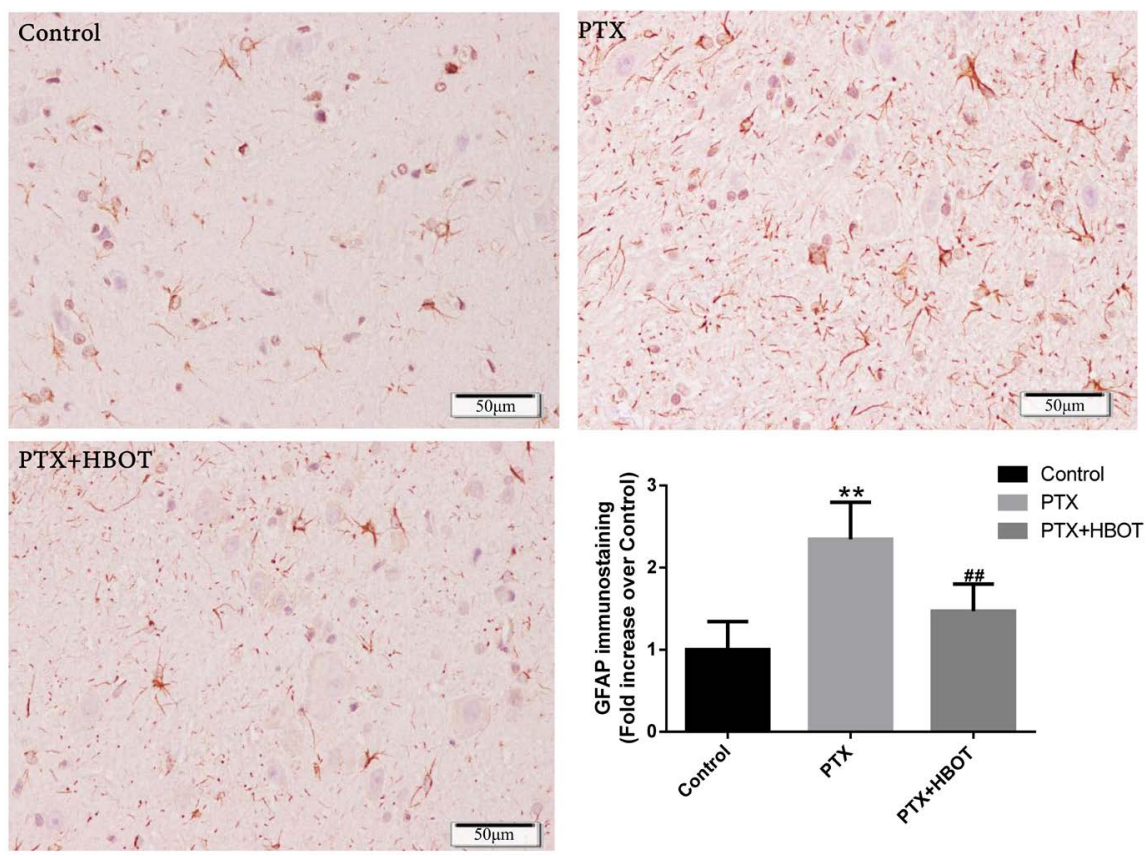

Figure 3. GFAP staining for astrocytes expression in the dorsal horn of the spinal cord among groups, $\mathrm{N}=5$ per group. ${ }^{*} \mathrm{P}<0.01$ vs. Control group, ${ }^{\# \#} \mathrm{P}<0.01$ vs. PTX group. 


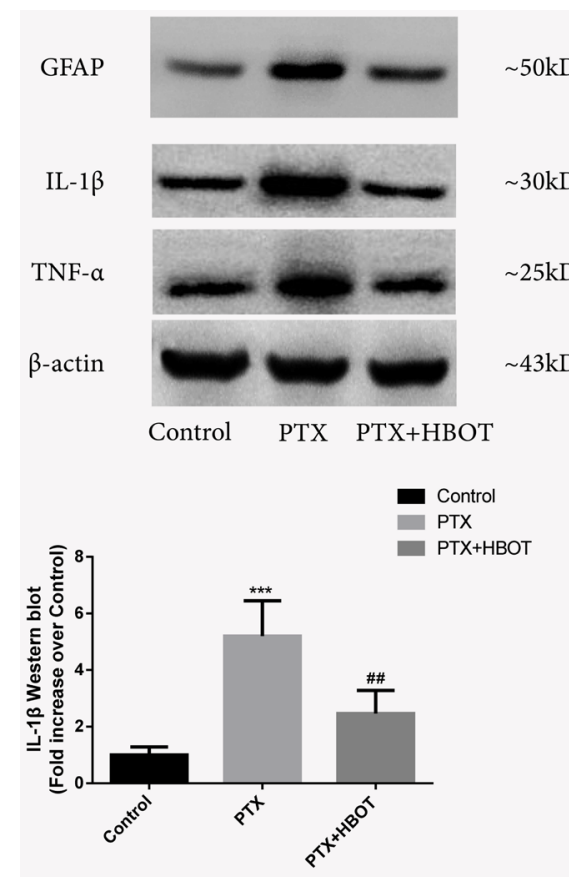

(b)

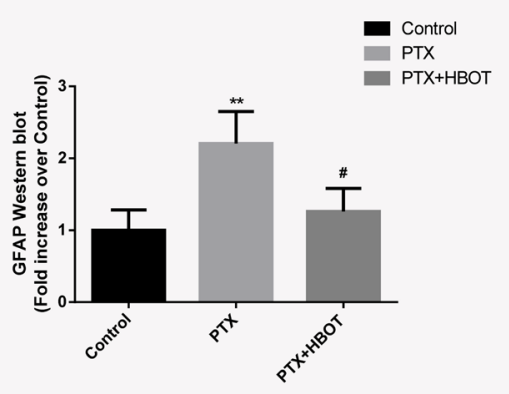

(a)

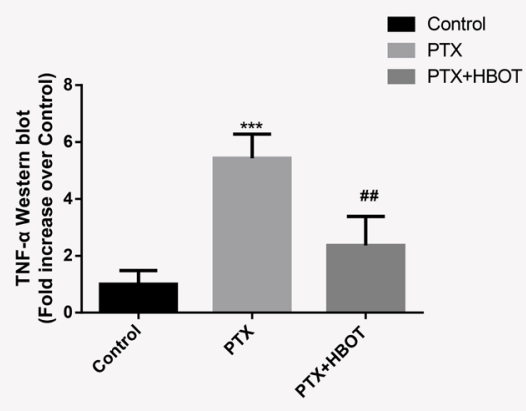

(c)

Figure 4. The expression of GFAP(a), IL-1 $\beta(\mathrm{b})$, TNF- $\alpha(\mathrm{c})$ in the dorsal horn of the spinal cord among groups, $\mathrm{N}=5$ per group. ${ }^{* *} \mathrm{P}<0.01,{ }^{* *} \mathrm{P}<0.001$ vs. Control group, ${ }^{*} \mathrm{P}<0.05$ ${ }^{\# \#} \mathrm{P}<0.01$ vs. PTX group.

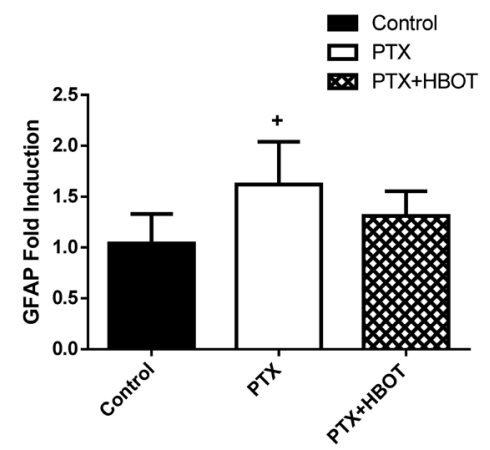

(a)

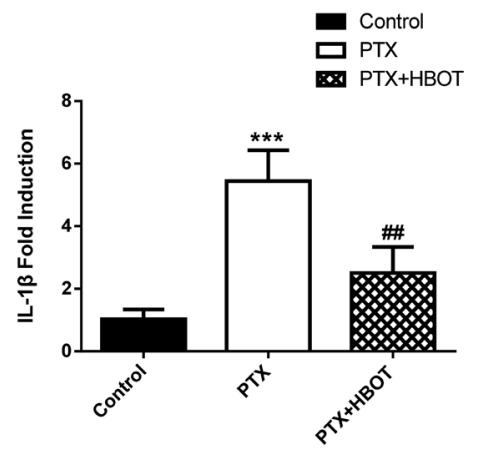

(b)

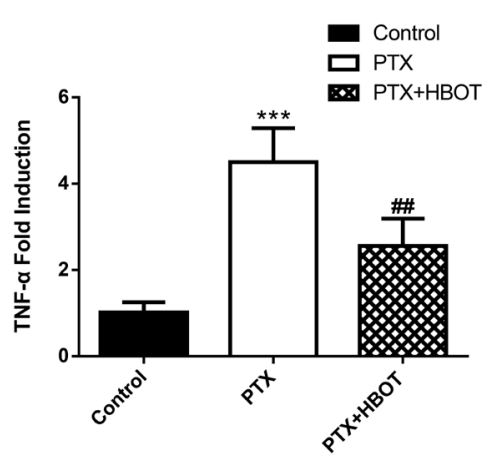

(c)

Figure 5. The mRNA levels of GFAP(a), IL-1 $\beta(\mathrm{b})$, TNF- $\alpha(\mathrm{c})$ in the dorsal horn of the spinal cord among groups, $\mathrm{N}=5$ per group. ${ }^{+} \mathrm{P}=0.0467$ vs. Control group, ${ }^{* * *} \mathrm{P}<0.001$ vs. Control group, ${ }^{\# \#} \mathrm{P}<0.01$ vs. PTX group. 


\section{Discussion}

The CIPNP model was established by paclitaxel which was widely used in the study of CIPNP, and had no significant influence on the health of rats [17] [18] [19]. However, few reported that the weight of rats slightly decreased at this dose [20]. In the present study, we demonstrated that there were no significant differences in weight changes among the groups during the experiment. Under the designed dose, two weeks after the first injection can cause stable symptoms of hyperalgesia, and reached the peak in about a month [13] [17] [21], and then relieved after four months [17] [21]. In the present study, stable symptoms of hyperalgesia were observed on the 14th day, and lasted until the rats were sacrificed on the 28th day, just as reported above. This period is an important stage in the development of CIPNP. So, the interventions were carried out during this period.

The analgesic effect produced by HBOT has two stages. The first stage is short-term analgesic effect. Due to different treatment parameters, this effect gradually disappears after several hours [22] [23]. Some studies reported that HBOT can produce short-term hyperalgesia symptoms and quickly fade into short-term analgesic effect [10]. This phenomenon may be due to a temporary increase of tissue reactive oxygen species [24]. The second stage is long-term analgesic effect. After continuous HBOT, the analgesic effect will last for up to 3 weeks [22]. This study focused on the long-term analgesic effect of HBOT and the analgesic effects gradually increased followed continuous treatment, and then decreased slightly after the end of the treatment for a week. These results fully indicate that HBOT can also be used to treat CIPNP with a certain long-term therapeutic effect, and this therapy has no significant impact on the health of rats.

The short-term effect of HBOT induced analgesia is related to the activation of opioid receptors [22] [25] [26] [27], while the long-term effect cannot be blocked by opioid receptor blockers [22]. The long-term efficacy of hyperbaric oxygen may be closely related to neuroinflammation mediated by glial cells in spinal cord [12]. This study showed that HBOT has a long-term effect on the regulation of spinal Astrocyte activation and Neuroinflammation after the cease of treatment for one week. This effect has been produced not only in a long term but also during HBOT [10] [11] [12]. In view of the long-term effect of HBOT, the expression of mRNA levels was detected. The results showed that the expression of mRNA levels of inflammatory factors was consistent with Western blot, but the mRNA expression of GFAP increased slightly after modeling. Because of the more stringent size of test of RT-PCR than Western blot $(\mathrm{p}<0.01$ or fold change $>2$ [28]), there was no statistical difference of GFAP between groups in this study. The inconsistency expression between mRNA and protein may be due to the process from gene to protein, such as gene transcriptional regulation, gene translational control, etc.

The upstream pathway of HBOT regulating the activation of spinal glial cells 
is related to reactive oxygen [29] and reactive nitrogen [12]. It has also been reported that HBOT has analgesic effect by regulating autophagy [30] [31] and apoptosis [32] [33] [34]. However, the reports above are limited to CCI and other neural injury models, but not to CIPNP. HBOT can enhance Chemosensitization [35], which indicates that HBOT is also a great method in the treatment of CIPNP. However, the inconsistency of mRNA and protein expression in astrocytes needs to be verified by more tests, and the reasons for that need to be further studied on the regulation of gene expression. In addition, we have found a pain regulatory system: the Endogenous cannabinoid system which can also treat CIPNP and has the ability to regulate glial cell activation [36]. It has been reported that the Cannabinoid type II receptor in spinal cord can regulate the activation of glial cells to mediate analgesia [37], and the type I receptor is also involved in the treatment of pain by regulating glial cells [7]. The relationship between $\mathrm{HBOT}$ and Endogenous cannabinoid system can be further explored based on the activation of glial cells. There's a high incidence of CIPNP and HBOT has been reported as neuroprotective [38]. So, the prevention of HBOT on CIPNP is also the research direction in the future.

\section{Acknowledgements}

This research was supported by China Postdoctoral Science Foundation (2016M602101) and Qingdao Postdoctoral Application Research Foundation (2016056).

\section{Conflicts of Interest}

The authors declare no conflicts of interest regarding the publication of this paper.

\section{References}

[1] Sisignano, M., Baron, R., Scholich, K., et al. (2014) Mechanism-Based Treatment for Chemotherapy-Induced Peripheral Neuropathic Pain. Nature Reviews Neurology, 10, 694-707. https://doi.org/10.1038/nrneurol.2014.211

[2] Miltenburg, N.C. and Boogerd, W. (2014) Chemotherapy-Induced Neuropathy: A Comprehensive Survey. Cancer Treatment Reviews, 40, 872-882. https://doi.org/10.1016/j.ctrv.2014.04.004

[3] Kidwell, K.M., Yothers, G., Ganz, P.A., et al. (2012) Long-Term Neurotoxicity Effects of Oxaliplatin Added to Fluorouracil and Leucovorin as Adjuvant Therapy for Colon Cancer: Results from National Surgical Adjuvant Breast and Bowel Project trials C-07 and LTS-01. Cancer, 118, 5614-5622. https://doi.org/10.1002/cncr.27593

[4] Pachman, D.R., Watson, J.C. and Loprinzi, C.L. (2014) Therapeutic Strategies for Cancer Treatment Related Peripheral Neuropathies. Current Treatment Options in Oncology, 15, 567-580. https://doi.org/10.1007/s11864-014-0303-7

[5] Sutherland, A.M., Clarke, H.A., Katz, J., et al. (2016) Hyperbaric Oxygen Therapy: A New Treatment for Chronic Pain? Pain Practice, 16, 620-628. https://doi.org/10.1111/papr.12312 
[6] Lees, J.G., Makker, P.G.S., Tonkin, R.S., et al. (2017) Immune-Mediated Processes Implicated in Chemotherapy-Induced Peripheral Neuropathy. European Journal of Cancer, 73, 22-29.

[7] Parvathy, S.S. and Masocha, W. (2015) Coadministration of Indomethacin and Minocycline Attenuates Established Paclitaxel-Induced Neuropathic Thermal Hyperalgesia: Involvement of Cannabinoid CB1 Receptors. Scientific Reports, 5, Article No. 10541. https://doi.org/10.1038/srep10541

[8] Ji, R.-R., Berta, T. and Nedergaard, M. (2013) Glia and Pain: Is Chronic Pain a Gliopathy? Pain, 154, S10-S28. https://doi.org/10.1016/j.pain.2013.06.022

[9] Zhou, Y.-Q., Liu, D.-Q., Chen, S.-P., et al. (2018) Minocycline as a Promising Therapeutic Strategy for Chronic Pain. Pharmacological Research, 134, 305-310. https://doi.org/10.1016/j.phrs.2018.07.002

[10] Zhao, B.S., Meng, L.X., Ding, Y.Y., et al. (2014) Hyperbaric Oxygen Treatment Produces an Antinociceptive Response Phase and Inhibits Astrocyte Activation and Inflammatory Response in a Rat Model of Neuropathic Pain. Journal of Molecular Neuroscience, 53, 251-261. https://doi.org/10.1007/s12031-013-0213-3

[11] Zhao, B., Pan, Y., Xu, H., et al. (2017) Hyperbaric Oxygen Attenuates Neuropathic Pain and Reverses Inflammatory Signaling Likely via the Kindlin-1/Wnt-10a Signaling Pathway in the Chronic pain Injury Model in Rats. The Journal of Headache and Pain, 18, 1. https://doi.org/10.1186/s10194-016-0713-y

[12] Ding, Y., Yao, P., Hong, T., et al. (2018) The Analgesic Effect of Early Hyperbaric Oxygen Treatment in Chronic Constriction Injury Rats and Its Influence on nNOS and iNOS Expression and Inflammatory Factor Production. Molecular Pain, 14, 1744806918765837. https://doi.org/10.1177/1744806918765837

[13] Polomano, R.C., Mannes, A.J., Clark, U.S., et al. (2001) A Painful Peripheral Neuropathy in the Rat Produced by the Chemotherapeutic Drug, Paclitaxel. Pain, 94, 293-304. https://doi.org/10.1016/S0304-3959(01)00363-3

[14] Chaplan, S.R., Bach, F.W., Pogrel, J.W., et al. (1994) Quantitative Assessment of Tactile Allodynia in the Rat Paw. Journal of Neuroscience Methods, 53, 55-63. https://doi.org/10.1016/0165-0270(94)90144-9

[15] Schmittgen, T.D. and Livak, K.J. (2008) Analyzing Real-Time PCR Data by the Comparative C(T) Method. Nature Protocols, 3, 1101-1108. https://doi.org/10.1038/nprot.2008.73

[16] Rahn, E.J., Deng, L., Thakur, G.A., et al. (2014) Prophylactic Cannabinoid Administration Blocks the Development of Paclitaxel-Induced Neuropathic Nociception during Analgesic Treatment and Following Cessation of Drug Delivery. Molecular Pain, 10, 27. https://doi.org/10.1186/1744-8069-10-27

[17] Griffiths, L.A., Duggett, N.A., Pitcher, A.L., et al. (2018) Evoked and Ongoing Pain-Like Behaviours in a Rat Model of Paclitaxel-Induced Peripheral Neuropathy. Pain Research \& Management, 2018, Article ID: 8217613.

[18] Segat, G.C., Manjavachi, M.N., Matias, D.O., et al. (2017) Antiallodynic Effect of $\beta$-Caryophyllene on Paclitaxel-Induced Peripheral Neuropathy in Mice. Neuropharmacology, 125, 207-219. https://doi.org/10.1016/j.neuropharm.2017.07.015

[19] Hwang, B.-Y., Kim, E.-S., Kim, C.-H., et al. (2012) Gender Differences in Paclitaxel-Induced Neuropathic Pain Behavior and Analgesic Response in Rats. Korean Journal of Anesthesiology, 62, 66-72. https://doi.org/10.4097/kjae.2012.62.1.66

[20] Cavaletti, G., Cavalletti, E., Montaguti, P., et al. (1997) Effect on the Peripheral Nervous System of the Short-Term Intravenous Administration of Paclitaxel in the 
Rat. Neurotoxicology, 18, 137-145.

[21] Flatters, S.J.L. and Bennett, G.J. (2006) Studies of Peripheral Sensory Nerves in Paclitaxel-Induced Painful Peripheral Neuropathy: Evidence for Mitochondrial Dysfunction. Pain, 122, 245-257. https://doi.org/10.1016/j.pain.2006.01.037

[22] Chung, E., Zelinski, L.M., Ohgami, Y., et al. (2010) Hyperbaric Oxygen Treatment Induces a 2-Phase Antinociceptive Response of Unusually Long Duration in Mice. The Journal of Pain, 11, 847-853. https://doi.org/10.1016/j.jpain.2009.12.004

[23] Zelinski, L.M., Ohgami, Y., Chung, E., et al. (2009) A Prolonged Nitric Oxide-Dependent, Opioid-Mediated Antinociceptive Effect of Hyperbaric Oxygen in Mice. The Journal of Pain, 10, 167-172. https://doi.org/10.1016/j.jpain.2008.08.003

[24] Thom, S.R. (2009) Oxidative Stress Is Fundamental to Hyperbaric Oxygen Therapy. Journal of Applied Physiology, 106, 988-995.

[25] Zhang, Y., Stolz, P.A., Shirachi, D.Y., et al. (2014) Reduced Antinociceptive Responsiveness to Hyperbaric Oxygen in Opioid-Tolerant Mice. European Journal of Pain, 18, 1032-1039. https://doi.org/10.1002/j.1532-2149.2013.00448.x

[26] Gibbons, C.R., Liu, S., Zhang, Y., et al. (2013) Involvement of Brain Opioid Receptors in the Anti-Allodynic Effect of Hyperbaric Oxygen in Rats with Sciatic Nerve Crush-Induced Neuropathic Pain. Brain Research, 1537, 111-116. https://doi.org/10.1016/j.brainres.2013.08.050

[27] Heeman, J.H., Zhang, Y., Shirachi, D.Y., et al. (2013) Involvement of Spinal Cord Opioid Mechanisms in the Acute Antinociceptive Effect of Hyperbaric Oxygen in Mice. Brain Research, 1540, 42-47. https://doi.org/10.1016/j.brainres.2013.09.050

[28] Xu, J., Diaz, P., Bie, B., et al. (2014) Spinal Gene Expression Profiling and Pathways Analysis of a CB2 Agonist (MDA7)-Targeted Prevention of Paclitaxel-Induced Neuropathy. Neuroscience, 260, 185-194. https://doi.org/10.1016/j.neuroscience.2013.12.028

[29] Xiao, W.H. and Bennett, G.J. (2012) Effects of Mitochondrial Poisons on the Neuropathic Pain Produced by the Chemotherapeutic Agents, Paclitaxel and Oxaliplatin. Pain, 153, 704-709. https://doi.org/10.1016/j.pain.2011.12.011

[30] Han, G., Liu, K., Li, L., et al. (2017) The Effects of Hyperbaric Oxygen Therapy on Neuropathic Pain via Mitophagy in Microglia. Molecular Pain, 13, 1744806917710862. https://doi.org/10.1177/1744806917710862

[31] Liu, Y.D., Wang, Z.B., Han, G., et al. (2017) Hyperbaric Oxygen Treatment Attenuates Neuropathic Pain by Elevating Autophagy Flux via Inhibiting mTOR Pathway. American Journal of Translational Research, 9, 2629-2638.

[32] Fu, H., Li, F., Thomas, S., et al. (2017) Hyperbaric Oxygenation Alleviates Chronic Constriction Injury (CCI)-Induced Neuropathic Pain and Inhibits GABAergic Neuron Apoptosis in the Spinal Cord. Scandinavian Journal of Pain, 17, 330-338. https://doi.org/10.1016/j.sjpain.2017.08.014

[33] Hu, Q., Fang, L., Li, F., et al. (2015) Hyperbaric Oxygenation Treatment Alleviates CCI-Induced Neuropathic Pain and Decreases Spinal Apoptosis. European Journal of Pain, 19, 920-928. https://doi.org/10.1002/ejp.618

[34] Zhao, B.S., Song, X.R., Hu, P.Y., et al. (2015) Hyperbaric Oxygen Treatment at Various Stages Following Chronic Constriction Injury Produces Different Antinociceptive Effects via Regulation of P2X4R Expression and Apoptosis. PLOS ONE, 10, e0120122. https://doi.org/10.1371/journal.pone.0120122

[35] Bennett, M.H., Feldmeier, J., Smee, R., et al. (2018) Hyperbaric Oxygenation for 
Tumour Sensitisation to Radiotherapy. The Cochrane Database of Systematic Reviews, 4, CD005007.

[36] O'Hearn, S., Diaz, P., Wan, B.A., et al. (2017) Modulating the Endocannabinoid Pathway as Treatment for Peripheral Neuropathic Pain: A Selected Review of Preclinical Studies. Annals of Palliative Medicine, 6, S209-S214.

https://doi.org/10.21037/apm.2017.08.04

[37] Maldonado, R., Baños, J.E. and Cabañero, D. (2016) The Endocannabinoid System and Neuropathic Pain. Pain, 157, S23-S32.

https://doi.org/10.1097/j.pain.0000000000000428

[38] Hu, Q., Manaenko, A., Matei, N., et al. (2016) Hyperbaric Oxygen Preconditioning: A Reliable Option for Neuroprotection. Medical Gas Research, 6, 20-32.

https://doi.org/10.4103/2045-9912.179337 